\title{
Biological screening of selected Pacific Northwest forest plants using the brine shrimp (Artemia salina) toxicity bioassay
}

Yvette M. Karchesy ${ }^{1}$, Rick G. Kelsey ${ }^{2 *}$, George Constantine ${ }^{3}$ and Joseph J. Karchesy ${ }^{1}$

\begin{abstract}
The brine shrimp (Artemia salina) bioassay was used to screen 211 methanol extracts from 128 species of Pacific Northwest plants in search of general cytotoxic activity. Strong toxicity $\left(\mathrm{LC}_{50}<100 \mu \mathrm{g} / \mathrm{ml}\right)$ was found for 17 extracts from 13 species, with highest activity observed for Angelica arguta roots at $<10 \mu \mathrm{g} / \mathrm{ml}$. Notably, four species of cedar trees and one of juniper in the family Cupressaceae dominated this group with $\mathrm{LC}_{50}$ for heartwood extracts ranging from 15 to $89 \mu \mathrm{g} / \mathrm{ml}$. Moderate toxicity $\left(\mathrm{LC}_{50} 100-500 \mu \mathrm{g} / \mathrm{ml}\right.$ ) was found in 38 extracts from 27 species, while weak toxicity $\left(\mathrm{LC}_{50} 500-1000 \mathrm{\mu g} / \mathrm{ml}\right)$ was detected for 17 extracts in 16 species. There were 139 extracts from 99 species that were non-toxic $\left(\mathrm{LC}_{50}>1000 \mathrm{\mu g} / \mathrm{ml}\right)$. Our subsequent studies of conifer heartwoods with strong activity confirm the assay's value for identifying new investigational leads for materials with insecticidal and fungicidal activity.
\end{abstract}

Keywords: Brine shrimp lethality, Artemia salina, Methanol extracts, Bioactivity

\section{Background}

The forests and rangelands of Washington and Oregon are diverse ecosystems ranging from the temperate rainforests of the Olympic Peninsula in Washington to the semiarid shrub-steppe of southeastern Oregon (Franklin and Dyrness 1988). Across this region, fir, pine and cedar species are basic foundations to industries producing lumber and structural wood products. Native Americans have long used many forest plants for foods, medicines and handmade materials to improve daily life (Gunther 1973; Forlines et al. 1992). There remains an interest in the herbal remedies (Moore 1993), and many of the plants still have potential for development of new, natural sources of medicines and insecticides.

The brine shrimp toxicity bioassay is a simple method of screening crude plant extracts for cytotoxicity (Meyer et al. 1982; McLaughlin et al. 1991) and is an indicator of potential antitumor, insecticidal, and fungicidal activity

\footnotetext{
${ }^{*}$ Correspondence: rkelsey@fs.fed.us

2 USDA Forest Service, Pacific Northwest Research Station, Corvallis, OR 97331, USA

Full list of author information is available at the end of the article
}

(Michael et al. 1956; Harwig and Scott 1971; McLaughlin et al. 1998). The mode of action causing toxicity is unknown, but the results typically correlate with more specific bioactivity tests. The brine shrimp bioassay has also been used to guide the isolation of bioactive compounds, testing of water quality, and detection of fungal toxins (Nguta et al. 2011; Arcanjo et al. 2012; Gadir 2012). This method is an attractive pre-screen for such activities as it is relatively simple and inexpensive to test large numbers of crude plant extracts in a relatively short time. Most surveys of this type have been carried out on traditional medicinal plants of various cultures from around the world (Pimentel et al. 2002; Krishnarajua et al. 2005; Rahman et al. 2008; Moshi et al. 2010; Ved et al. 2010; Bussmann et al. 2011; Nguta et al. 2011; Oryema et al. 2011; Arcanjo et al. 2012; Gadir 2012; Nguta et al. 2012; Biradi and Hullatti 2014; Khatun et al. 2014). A few studies have targeted forest and savannah plants (Horgen et al. 2001; Adouom 2009; Rizwana et al. 2010; Soonthornchareonnon et al. 2012; Ravikumar et al. 2014).

In this paper we report survey results for some forest plants from the Pacific Northwest to gain a preliminary understanding of which ones may merit further, more 
specific testing with potential for developing new medicines and pesticides to benefit future generations.

\section{Methods}

\section{Plant materials}

Plants were collected during their active growing seasons in western Washington, western and central Oregon. Voucher specimens were deposited at the Oregon State University Herbarium.

\section{Preparation of extracts}

Plant materials were air-dried, ground and then extracted at room temperature for $48 \mathrm{~h}$ with methanol. The methanol was analytical grade and freshly distilled prior to use. Extracts were evaporated under vacuum on a rotary evaporator and the residue briefly freeze dried under high vacuum to remove traces of solvent and water, then stored at $-20{ }^{\circ} \mathrm{C}$ until tested.

\section{Brine shrimp toxicity bioassay}

Bioassays of the crude extracts were carried out as described by Meyer et al. (1982) and McLaughlin et al. (1991) on freshly hatched brine shrimp (Artemia salina Leach). Triplicate samples of each extract were tested initially at concentrations of 10, 100 and $1000 \mathrm{ppm}(\mu \mathrm{g} / \mathrm{mL})$ in vials containing $5 \mathrm{~mL}$ of brine solution and 10 shrimp. Survivors were counted after $24 \mathrm{~h}$ and the median lethal concentration $\left(\mathrm{LC}_{50}\right)$ with $95 \%$ confidence intervals calculated using Probit Analysis.

\section{Results}

Results of the brine shrimp cytotoxicity screening are shown in Table 1 . Extracts with $\mathrm{LC}_{50}$ values $>1000 \mu \mathrm{g} /$ $\mathrm{ml}$ are considered non-toxic (Meyer et al. 1982). Values between 500 and $1000 \mu \mathrm{g} / \mathrm{ml}$ are considered weakly toxic, those between 100 and $500 \mu \mathrm{g} / \mathrm{ml}$ as moderately toxic, and those $<100 \mu \mathrm{g} / \mathrm{ml}$ as strongly toxic (Nguta et al. 2012). A total of 211 crude methanol extracts from 128 species, 116 genera, and 49 families are represented. Strong cytotoxic activity was found in 17 extracts from 13 species (Table 2), moderate toxicity in 38 extracts from 27 species, weak activity for 17 extracts in 16 species, and
139 non-toxic extracts from 99 species. The proportions of all extracts by activity category are shown in Fig. 1.

\section{Discussion}

There were more than twice as many extracts with moderate activity than there were with strong activity. Moderately active extracts need not be dismissed as unimportant, since Bussmann et al. (2011), Nguta et al. (2012) and others have noted that toxicity can vary significantly due to harvest time, collection location, plant organ or tissue, and solvent used for extraction. Alcohol or organic solvent extracts are often more toxic than aqueous ones, but not always. Extracts from genera and species with the strongest bioactivity can also exhibit a wide range in their levels of activity for the same reasons, thus varying among experiments and research groups. Given this natural variability and our extensive list of genera and species we decided not to attempt cross comparing levels of activity with those observed by others, as it is beyond the scope of this report.

Tissues identified with $\mathrm{LC}_{50}<100 \mu \mathrm{g} / \mathrm{ml}$ cytotoxicity have served us as leads for further studies of bioactive extracts and compounds from heartwoods of yellow, incense, and Port-Orford cedars, and western juniper against mosquitoes (Aedes aegypti), ticks (Ixodes scapularis), fleas (Xenopsylla cheopis) or microbes influencing animal and forest health (Johnston et al. 2001; Panella et al. 2005; Dietrich et al. 2006; Manter et al. 2006, 2007; Dolan et al. 2007, 2009). It is worthwhile noting that three of the compounds in yellow or incense cedar heartwoods have different modes of action than other commercially available mosquito adulticides currently in use (McAllister and Adams 2010). New modes of action are particularly relevant in the search for compounds to overcome resistance to existing pesticides.

\section{Conclusion}

Natural products from Pacific Northwest forest resources can offer alternative biocides and repellent compounds with activities comparable to synthetic pesticides for control of arthropods of public health concern and forest microbial pathogens. Other bioactive extracts from our 
Table 1 Brine shrimp toxicity at $24 \mathrm{~h}$ exposure to plant extracts

\begin{tabular}{|c|c|c|c|c|}
\hline Plant family and species & Common name & Part used & $\mathrm{LC}_{50}(\mu \mathrm{g} / \mathrm{ml})$ & $95 \% \mathrm{Cl}$ \\
\hline \multicolumn{5}{|l|}{ Aceraceae } \\
\hline \multirow[t]{2}{*}{ Acer circinatum } & Vine maple & Bark & $>1000$ & \\
\hline & & Leaves & $>1000$ & \\
\hline \multirow[t]{2}{*}{ Acer macrophyllum } & Big leaf maple & Bark & $>1000$ & \\
\hline & & Catkins & $>1000$ & \\
\hline \multicolumn{5}{|l|}{ Adoxaceae } \\
\hline \multirow[t]{2}{*}{ Sambucus nigra subsp. caerulea } & Blue elderberry & Bark & $>1000$ & \\
\hline & & Berries & $>1000$ & \\
\hline Sambucus racemosa & Red elderberry & Bark & $>1000$ & \\
\hline Viburnum ellipticum & Oregon viburnum & Leaves & $>1000$ & \\
\hline \multicolumn{5}{|l|}{ Amaranthaceae } \\
\hline Amaranthus retroflexus & Pigweed & Aerial parts & $>1000$ & \\
\hline \multicolumn{5}{|l|}{ Apocynaceae } \\
\hline Apocynum androsaemifolium & Spreading dogbane & Aerial parts & 88 & $55-141$ \\
\hline \multicolumn{5}{|l|}{ Araceae } \\
\hline \multirow[t]{3}{*}{ Lysichiton americanus } & Skunk cabbage & Flowers & $>1000$ & \\
\hline & & Leaves & $>1000$ & \\
\hline & & Roots & $>1000$ & \\
\hline \multicolumn{5}{|l|}{ Araliaceae } \\
\hline \multirow[t]{6}{*}{ Oplopanax horridum } & Devil's club & Berries-green & 338 & $292-573$ \\
\hline & & Berries-red & 239 & $187-279$ \\
\hline & & Leaves & $>1000$ & \\
\hline & & Petioles & 237 & $153-372$ \\
\hline & & Root bark & 21 & $13-32$ \\
\hline & & Stem bark & 35 & $23-51$ \\
\hline \multicolumn{5}{|l|}{ Aristolochiaceae } \\
\hline Asarum caudatum & Wild ginger & Aerial parts & 565 & $364-918$ \\
\hline \multicolumn{5}{|l|}{ Aquifoliaceae } \\
\hline Ilex aquifolium & Holly & Leaves & $>1000$ & \\
\hline \multicolumn{5}{|l|}{ Berberidaceae } \\
\hline \multirow[t]{3}{*}{ Berberis aquifolium } & Tall Oregon grape & Berries green & 305 & $245-352$ \\
\hline & & Berry stems & $>1000$ & \\
\hline & & Flower heads & 608 & $404-4630$ \\
\hline \multirow[t]{2}{*}{ Berberis nervosa } & Cascade Oregon grape & Leaves & $>1000$ & \\
\hline & & Roots & $>1000$ & \\
\hline Berberis repens & Low Oregon grape & Leaves & $>1000$ & \\
\hline \multicolumn{5}{|l|}{ Betulaceae } \\
\hline \multirow[t]{2}{*}{ Alnus rubra } & Red alder & Bark & $>1000$ & \\
\hline & & Leaves & $>1000$ & \\
\hline Corylus cornuta & Hazelnut & Bark & $>1000$ & \\
\hline \multicolumn{5}{|l|}{ Boraginaceae } \\
\hline Mertensia paniculata & Tall bluebell & Aerial parts & $>1000$ & \\
\hline Myosotis laxa & Small flowered forget-me-not & Aerial parts & $>1000$ & \\
\hline Symphytum officinale & Comfrey & Aerial parts & $>1000$ & \\
\hline \multicolumn{5}{|l|}{ Caprifoliaceae } \\
\hline \multirow[t]{2}{*}{ Lonicera involucrata } & Black twin-berry & Leaves & $>1000$ & \\
\hline & & Bark & $>1000$ & \\
\hline \multirow[t]{2}{*}{ Symphoricarpos albus } & Snowberry & Berries & $>1000$ & \\
\hline & & Leaves & $>1000$ & \\
\hline
\end{tabular}


Table 1 continued

\begin{tabular}{|c|c|c|c|c|}
\hline Plant family and species & Common name & Part used & $\mathrm{LC}_{50}(\mu \mathrm{g} / \mathrm{ml})$ & $95 \% \mathrm{Cl}$ \\
\hline \multicolumn{5}{|l|}{ Chenopodiaceae } \\
\hline Sarcocornia perennis & Pickleweed & Leaves & $>1000$ & \\
\hline \multicolumn{5}{|l|}{ Compositae (Asteraceae) } \\
\hline \multirow[t]{3}{*}{ Achillea millefolium } & Yarrow & Aerial parts & 565 & $364-918$ \\
\hline & & Leaves only & 300 & $216-402$ \\
\hline & & Seeds & $>1000$ & \\
\hline Ambrosia chamissonis & Silver burweed & Aerial parts & $>1000$ & \\
\hline Anaphalis margaritacea & Pearly everlasting & Aerial parts & 808 & $403-2800$ \\
\hline Antennaria geyeri & Pussy toes (Geyer) & Aerial parts & $>1000$ & \\
\hline \multirow[t]{2}{*}{ Anthemis cotula } & Dog fennel & Aerial parts & 246 & $182-320$ \\
\hline & & Roots & $>1000$ & \\
\hline Bellis perennis & Bellis (English daisy) & Aerial parts & 454 & $282-760$ \\
\hline \multirow[t]{2}{*}{ Centaurea xmoncktonii } & Meadow & Aerial parts & 277 & $203-355$ \\
\hline & knapweed & Roots & 109 & $96-152$ \\
\hline \multirow[t]{2}{*}{ Centaurea solstitialis } & Yellow star- & Aerial parts & $>1000$ & \\
\hline & thistle & Roots & 693 & $423-1349$ \\
\hline \multirow[t]{2}{*}{ Centaurea stoebe subsp. micranthos } & Spotted knapweed & Aerial parts & $>1000$ & \\
\hline & & Roots & 87 & $56-135$ \\
\hline Chrysothamnus viscidiflorus & Rabbit brush (Green) & Aerial parts & $>1000$ & \\
\hline Cichorium intybus & Chicory & Aerial parts & $>1000$ & \\
\hline Cirsium vulgare & Bull thistle & Aerial parts & $>1000$ & \\
\hline Conyza canadensis & Horseweed & Aerial parts & 159 & $96-267$ \\
\hline Ericameria nauseosa & Rabbit brush (Gray) & Aerial parts & 579 & $360-1006$ \\
\hline Eriophyllum lanatum & Woolly sunshine & Aerial parts & $>1000$ & \\
\hline \multirow[t]{2}{*}{ Grindelia integrifolia } & Gumweed & Aerial parts & 173 & $107-276$ \\
\hline & & Roots & 99 & $75-116$ \\
\hline Hypochaeris glabra & Cat's ear & Aerial parts & $>1000$ & \\
\hline Lapsana communis & Nipplewort & Aerial parts & $>1000$ & \\
\hline \multirow[t]{2}{*}{ Leucanthemum vulgare } & Oxeye daisy & Aerial parts & 16 & $10-25$ \\
\hline & & Roots & 164 & $139-183$ \\
\hline Madia sativa & Tarweed & Aerial parts & $>1000$ & \\
\hline Matricaria discoidea & Pineapple weed & Aerial parts & 192 & $160-208$ \\
\hline Senecio jacobaea & Tansy ragwort & Aerial parts & $>1000$ & \\
\hline Solidago canadensis & Canada goldenrod & Aerial parts & 827 & $458-2214$ \\
\hline \multirow[t]{2}{*}{ Sonchus asper } & Prickly sow & Leaves & $>1000$ & \\
\hline & thistle & Roots & $>1000$ & \\
\hline Symphyotrichum subspicatum & Douglas aster & Aerial parts & $>1000$ & \\
\hline Tanacetum vulgare & Common tansy & Aerial parts & 62 & $39-93$ \\
\hline Tragopogon porrifolius & Salsify & Aerial parts & $>1000$ & \\
\hline \multicolumn{5}{|l|}{ Convolvulaceae } \\
\hline Convolvulus arvensis & Orchard morning glory & Aerial parts & $>1000$ & \\
\hline \multicolumn{5}{|l|}{ Cornaceae } \\
\hline Cornus nuttallii & Dogwood & Bark & $>1000$ & \\
\hline \multicolumn{5}{|l|}{ Cupressaceae } \\
\hline \multirow[t]{5}{*}{ Callitropsis nootkatensis } & Yellow-cedar & Foliage & 42 & $27-65$ \\
\hline & & Heartwood & 89 & $53-114$ \\
\hline & & Outer Bark & 693 & $423-1349$ \\
\hline & & Inner Bark & 15 & $8-24$ \\
\hline & & Sapwood & $>1000$ & \\
\hline
\end{tabular}


Table 1 continued

\begin{tabular}{|c|c|c|c|c|}
\hline Plant family and species & Common name & Part used & $\mathrm{LC}_{50}(\mu \mathrm{g} / \mathrm{ml})$ & $95 \% \mathrm{Cl}$ \\
\hline \multirow[t]{2}{*}{ Calocedrus decurrens } & Incense cedar & Heartwood & 55 & $35-80$ \\
\hline & & Sapwood & $>1000$ & \\
\hline \multirow[t]{2}{*}{ Cedrus deodara ${ }^{1}$} & Deodar cedar & Heartwood & 15 & $9-24$ \\
\hline & & Sapwood & 36 & $30-39$ \\
\hline Chamaecyparis lawsoniana & Port Orford cedar & Heartwood & 31 & $23-39$ \\
\hline \multirow[t]{2}{*}{$\times$ Hesperotropsis leylandii } & Leyland cypress & Heartwood & 118 & $81-161$ \\
\hline & & Sapwood & $>1000$ & \\
\hline \multirow[t]{6}{*}{ Juniperus occidentalis } & Juniper & Berries & $>1000$ & \\
\hline & (Western) & Leaves & $>1000$ & \\
\hline & & Heartwood & 66 & $56-77$ \\
\hline & & Inner Bark & $>1000$ & \\
\hline & & Outer Bark & $>1000$ & \\
\hline & & Sapwood & 189 & $116-338$ \\
\hline \multicolumn{5}{|l|}{ Elaeagnaceae } \\
\hline \multirow[t]{4}{*}{ Shepherdia canadensis } & Soapberry & Berries & 387 & $255-571$ \\
\hline & & Leaves & $>1000$ & \\
\hline & & Leaves with twigs & $>1000$ & \\
\hline & & Outer Bark & 314 & $174-662$ \\
\hline \multicolumn{5}{|l|}{ Ericaceae } \\
\hline \multirow[t]{2}{*}{ Arbutus menziesii } & Pacific madrone & Inner Bark & $>1000$ & \\
\hline & & Red berries & $>1000$ & \\
\hline \multirow[t]{2}{*}{ Arctostaphylos columbiana } & Hairy manzanita & Bark & $>1000$ & \\
\hline & & Leaves & $>1000$ & \\
\hline Arctostaphylos patula & Green leaf manzanita & Aerial parts & $>1000$ & \\
\hline \multirow[t]{2}{*}{ Arctostaphylos uva-ursi } & Kinnikinnick & Berries-red Leaves/stems & $>1000$ & \\
\hline & & & $>1000$ & \\
\hline \multirow[t]{2}{*}{ Chimaphila umbellata } & Prince's pine & Aerial parts & 155 & $131-177$ \\
\hline & & Stems & 126 & $86-170$ \\
\hline Gaultheria shallon & Salal & Leaves & $>1000$ & \\
\hline \multirow[t]{2}{*}{ Rhododendron macrophyllum } & Pacific rhododendron & Bark & $>1000$ & \\
\hline & & Leaves & $>1000$ & \\
\hline \multicolumn{5}{|l|}{ Fagaceae } \\
\hline \multirow[t]{4}{*}{ Quercus garryana } & White oak & Galls & $>1000$ & \\
\hline & & Heartwood & 301 & $195-468$ \\
\hline & & Inner Bark & $>1000$ & \\
\hline & & Leaves & $>1000$ & \\
\hline \multicolumn{5}{|l|}{ Fumariaceae } \\
\hline Dicentra formosa & Wild bleeding heart & Aerial parts & $>1000$ & \\
\hline \multicolumn{5}{|l|}{ Geraniaceae } \\
\hline Geranium dissectum & Cut-leaf geranium & Aerial parts & $>1000$ & \\
\hline \multicolumn{5}{|l|}{ Iridaceae } \\
\hline Iris tenax & Oregon iris & Aerial parts & $>1000$ & \\
\hline \multicolumn{5}{|l|}{ Labiatae } \\
\hline Prunella vulgaris & Heal all; Self-heal & Aerial parts & $>1000$ & \\
\hline Stachys cooleyae & $\begin{array}{l}\text { Cooley's hedge nettle (False stinging } \\
\text { nettle) }\end{array}$ & Aerial parts & $>1000$ & \\
\hline \multicolumn{5}{|l|}{ Lauraceae } \\
\hline \multirow[t]{2}{*}{ Umbellularia californica } & Oregon myrtle & Heartwood & 363 & $255-488$ \\
\hline & & Sapwood & $>1000$ & \\
\hline
\end{tabular}


Table 1 continued

\begin{tabular}{|c|c|c|c|c|}
\hline Plant family and species & Common name & Part used & $\mathrm{LC}_{50}(\mu \mathrm{g} / \mathrm{ml})$ & $95 \% \mathrm{Cl}$ \\
\hline \multicolumn{5}{|l|}{ Leguminosae } \\
\hline Cytisus scoparius & Scotch broom & Aerial parts & $>1000$ & \\
\hline \multirow[t]{2}{*}{ Dalea ornata } & Prairie clover & Aerial parts & 157 & $95-257$ \\
\hline & & Roots & 313 & $121-1632$ \\
\hline Robinia pseudoacacia & Black locust & Heartwood & $>1000$ & \\
\hline Trifolium pratense & Red clover & Aerial parts & $>1000$ & \\
\hline \multicolumn{5}{|l|}{ Liliaceae } \\
\hline \multirow[t]{3}{*}{ Camassia quamash } & Camas & Aerial parts & 212 & 150-952 \\
\hline & & Flowers & 272 & $148-583$ \\
\hline & & Leaves & 446 & 256-905 \\
\hline Prosartes smithii & Smith's fairy bell & Aerial parts & $>1000$ & \\
\hline \multicolumn{5}{|l|}{ Malvaceae } \\
\hline Malva neglecta & Dwarf mallow & Aerial parts & $>1000$ & \\
\hline \multicolumn{5}{|l|}{ Nyctaginaceae } \\
\hline Abronia latifolia & Yellow sandverbena & Aerial parts & $>1000$ & \\
\hline \multicolumn{5}{|l|}{ Onagraceae } \\
\hline Chamerion angustifolium & Fireweed & Aerial parts & $>1000$ & \\
\hline \multicolumn{5}{|l|}{ Oxalidaceae } \\
\hline Oxalis oregana & Oxalis & Aerial parts & 281 & $268-298$ \\
\hline \multicolumn{5}{|l|}{ Pinaceae } \\
\hline \multirow[t]{2}{*}{ Abies grandis } & Grand-fir & Needles (new) & $>1000$ & \\
\hline & & Needles (old) & $>1000$ & \\
\hline Picea sitchensis & Sitka spruce & Needles & $>1000$ & \\
\hline \multirow[t]{2}{*}{ Pinus monticola } & Western white & Bark & $>1000$ & \\
\hline & pine & Needles & 504 & $397-662$ \\
\hline \multirow[t]{4}{*}{ Pinus ponderosa } & Ponderosa pine & Bark & $>1000$ & \\
\hline & & Heartwood & 107 & 69-166 \\
\hline & & Needles & $>1000$ & \\
\hline & & Sapwood & $>1000$ & \\
\hline \multirow[t]{5}{*}{ Pseudotsuga menziesii } & Douglas-fir & Cones-green & $>1000$ & \\
\hline & & Heartwood & 663 & $422-1153$ \\
\hline & & Needles & $>1000$ & \\
\hline & & Outer bark & $>1000$ & \\
\hline & & Sapwood & $>1000$ & \\
\hline \multirow[t]{3}{*}{ Tsuga heterophylla } & Western & Cones-green & $>1000$ & \\
\hline & hemlock & Needles & $>1000$ & \\
\hline & & Sapwood & $>1000$ & \\
\hline \multicolumn{5}{|l|}{ Plantaginaceae } \\
\hline Plantago spp. & Plantain & Aerial parts & $>1000$ & \\
\hline \multicolumn{5}{|l|}{ Polygonaceae } \\
\hline Rumex spp. & Dock & Roots & 923 & $822-1537$ \\
\hline \multicolumn{5}{|l|}{ Polypodiaceae } \\
\hline Polypodium glycyrrhiza & Licorice fern & Roots & $>1000$ & \\
\hline \multirow[t]{2}{*}{ Polystichum munitum } & Sword fern & Leaves & $>1000$ & \\
\hline & & Roots & $>1000$ & \\
\hline Pteridium aquilinum & Bracken fern & Roots & $>1000$ & \\
\hline \multicolumn{5}{|l|}{ Portulacaceae } \\
\hline Claytonia sibirica & Siberian miners'lettuce & Aerial parts & $>1000$ & \\
\hline \multicolumn{5}{|l|}{ Primulaceae } \\
\hline Trientalis latifolia & Western starflower & Aerial parts & 539 & $430-627$ \\
\hline
\end{tabular}


Table 1 continued

\begin{tabular}{|c|c|c|c|c|}
\hline Plant family and species & Common name & Part used & $\mathrm{LC}_{50}(\mu \mathrm{g} / \mathrm{ml})$ & $95 \% \mathrm{Cl}$ \\
\hline \multicolumn{5}{|l|}{ Ranunculaceae } \\
\hline Clematis vitalba & Clematis & Aerial parts & $>1000$ & \\
\hline Delphinium trolliifolium & Delphinium & Aerial parts & 304 & $190-489$ \\
\hline Ranunculus occidentalis & Western buttercup & Aerial parts & $>1000$ & \\
\hline Ranunculus repens & Creeping buttercup & Aerial parts & $>1000$ & \\
\hline \multicolumn{5}{|l|}{ Rhamnaceae } \\
\hline \multirow[t]{2}{*}{ Rhamnus purshiana } & Cascara & Bark & 393 & $237-698$ \\
\hline & & Leaves & 247 & $186-667$ \\
\hline \multicolumn{5}{|l|}{ Rosaceae } \\
\hline \multirow[t]{3}{*}{ Aruncus dioicus } & Goat's beard & Flowers & $>1000$ & \\
\hline & & Leaves & $>1000$ & \\
\hline & & Roots & $>1000$ & \\
\hline \multirow[t]{2}{*}{ Crataegus douglasii } & Black hawthorn & Berries-green & $>1000$ & \\
\hline & & Leaves & $>1000$ & \\
\hline \multirow[t]{3}{*}{ Holodiscus discolor } & Ocean spray & Bark & $>1000$ & \\
\hline & & Flowers & $>1000$ & \\
\hline & & Leaves & $>1000$ & \\
\hline Malus fusca & Crabapple & Bark & $>1000$ & \\
\hline \multirow[t]{2}{*}{ Oemleria cerasiformis } & Indian-plum & Bark & $>1000$ & \\
\hline & & Stems + leaves + berries & $>1000$ & \\
\hline Potentilla pacifica & Pacific silverweed & Leaves & 632 & 298-2309 \\
\hline \multirow[t]{3}{*}{ Prunus spp. } & Cherry & Leaves & $>1000$ & \\
\hline & & Inner Bark & $>1000$ & \\
\hline & & Outer Bark & 490 & $354-614$ \\
\hline \multirow[t]{3}{*}{ Purshia tridentata } & Bitter-brush & Leaves & 870 & $533-1857$ \\
\hline & & Roots & 691 & $545-884$ \\
\hline & & Seeds & 144 & $101-192$ \\
\hline \multirow[t]{2}{*}{ Rosa nutkana } & Nootka rose & Leaves & $>1000$ & \\
\hline & & Stems & $>1000$ & \\
\hline Rubus parviflorus & Thimbleberry & Leaves & $>1000$ & \\
\hline \multirow[t]{2}{*}{ Rubus spectabilis } & Salmonberry & Bark & $>1000$ & \\
\hline & & Leaves & $>1000$ & \\
\hline Rubus ursinus & Blackberry (trailing) & Aerial parts & $>1000$ & \\
\hline \multirow[t]{2}{*}{ Sorbus scopulina } & Mountain ash & Berries & 318 & $308-328$ \\
\hline & & Leaves & $>1000$ & \\
\hline Spiraea douglasii & Spirea & Aerial parts & $>1000$ & \\
\hline \multicolumn{5}{|l|}{ Rubiaceae } \\
\hline Galium aparine & Cleavers & Aerial parts & $>1000$ & \\
\hline \multicolumn{5}{|l|}{ Salicaceae } \\
\hline Populus spp. & Cottonwood & Outer Bark & $>1000$ & \\
\hline \multicolumn{5}{|l|}{ Saxifragaceae } \\
\hline Tellima grandiflora & Fringecup & Aerial parts & $>1000$ & \\
\hline \multicolumn{5}{|l|}{ Scrophulariaceae } \\
\hline Digitalis purpurea & Foxglove & Aerial parts & $>1000$ & \\
\hline \multirow[t]{2}{*}{ Verbascum thapsus } & Common mullein & Aerial parts & $>1000$ & \\
\hline & & Roots & $>1000$ & \\
\hline \multicolumn{5}{|l|}{ Solanaceae } \\
\hline Solanum nigrum & Black nightshade & Aerial parts & 662 & $422-1153$ \\
\hline Taxaceae & & & & \\
\hline
\end{tabular}


Table 1 continued

\begin{tabular}{|c|c|c|c|c|}
\hline Plant family and species & Common name & Part used & $\mathrm{LC}_{50}(\mu \mathrm{g} / \mathrm{ml})$ & $95 \% \mathrm{Cl}$ \\
\hline Taxus brevifolia & Pacific yew & Heartwood & $>1000$ & \\
\hline \multicolumn{5}{|l|}{ Taxodiaceae } \\
\hline \multirow[t]{2}{*}{ Sequoiadendron giganteum } & Giant sequoia & Needles & 713 & $580-878$ \\
\hline & & Heartwood & 206 & $166-246$ \\
\hline \multicolumn{5}{|l|}{ Umbelliferae } \\
\hline \multirow[t]{2}{*}{ Angelica arguta } & Sharptooth angelica & Aerial parts & 123 & $94-371$ \\
\hline & & Roots & $<10$ & $--^{2}$ \\
\hline Daucus carota & Queen Anne's lace & Aerial parts & $>1000$ & \\
\hline Foeniculum vulgare & Fennel & Aerial parts & $>1000$ & \\
\hline \multirow[t]{2}{*}{ Heracleum maximum } & Cow parsnip & Roots & 249 & $167-384$ \\
\hline & & Umbels & 404 & $307-496$ \\
\hline Oenanthe sarmentosa & Pacific water parsley & Aerial parts & 76 & $48-117$ \\
\hline \multicolumn{5}{|l|}{ Urticaceae } \\
\hline \multirow[t]{2}{*}{ Urtica dioica } & Stinging nettle & Aerial parts & $>1000$ & \\
\hline & & Roots & $>1000$ & \\
\hline
\end{tabular}

1 Endemic to the Indian subcontinent, collected from a tree farm in Oregon

$210 \mu \mathrm{g} / \mathrm{ml}$ was the lowest concentration tested with mean mortality at $90 \%$

Table 2 Plant species and tissues with strong, $<100 \mu \mathrm{g} /$ $\mathrm{ml} \mathrm{LC}{ }_{50}$, brine shrimp toxicity at $24 \mathrm{~h}$ exposure to plant extracts

\begin{tabular}{llcl}
\hline Species & Part used & LC $_{\mathbf{5 0}}(\mathbf{\mu g} / \mathbf{m l})$ & $\mathbf{9 5} \mathbf{~ C l}$ \\
\hline Apocynum androsaemifolium & Aerial parts & 88 & $55-141$ \\
Oplopanax horridum & Root bark & 21 & $13-32$ \\
& Stem bark & 35 & $23-51$ \\
Centaurea stoebe subsp. & Roots & 87 & $56-135$ \\
micranthos & & & \\
Grindelia integrifolia & Roots & 99 & $75-116$ \\
Leucanthemum vulgare & Aerial parts & 16 & $10-25$ \\
Tanacetum vulgare & Aerial parts & 62 & $39-93$ \\
Callitropsis nootkatensis & Foliage & 42 & $27-65$ \\
& Heartwood & 89 & $53-114$ \\
Calocedrus decurrens & Inner bark & 15 & $8-24$ \\
Cedrus deodara' & Heartwood & 55 & $35-80$ \\
& Heartwood & 15 & $9-24$ \\
Chamaecyparis lawsoniana & Sapwood & 36 & $30-39$ \\
Juniperus occidentalis & Heartwood & 31 & $23-39$ \\
Angelica arguta & Heartwood & 66 & $56-77$ \\
Oenanthe sarmentosa & Roots & $<10$ & -2 \\
\hline Tnderial parts & 76 & $48-117$ \\
\hline
\end{tabular}

1 Endemic to the Indian subcontinent, collected from a tree farm in Oregon

$210 \mu \mathrm{g} / \mathrm{ml}$ was the lowest concentration tested with mean mortality at $90 \%$

brine shrimp screening need to be investigated further. In addition, other forest plants from this region need to be pre-screened by this method as well to provide a more

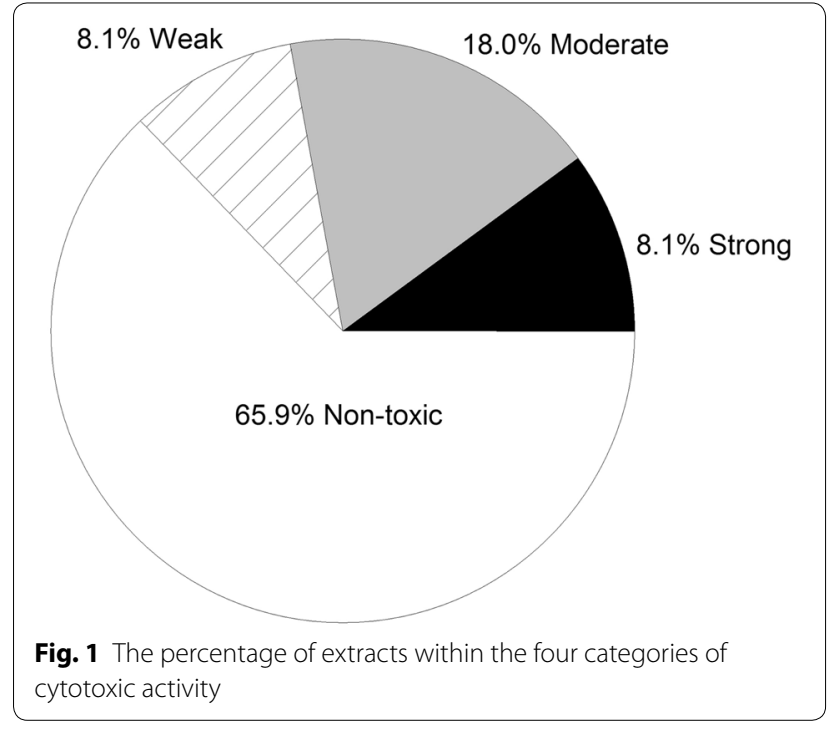

complete understanding of the potential value for all our forest and rangeland resources.

\section{Authors' contributions}

YMK collected plant material, prepared extracts, conducted the bioassays and processed the data. RGK collected some plants, prepared some extracts and co-wrote the manuscript. GC assisted with the bioassays. JJK conceived the study, collected some of the plants, and co-wrote the manuscript. All authors read and approved the final manuscript.

\section{Author details}

${ }^{1}$ Wood Science and Engineering, Oregon State University, Corvallis, OR 97331, USA. ${ }^{2}$ USDA Forest Service, Pacific Northwest Research Station, Corvallis, OR 
97331, USA. ${ }^{3}$ College of Pharmacy, Oregon State University, Corvallis, OR 97331, USA

\section{Acknowledgements}

The authors thank Richard Halse, Oregon State University Herbarium, for assistance with plant identification.

\section{Competing interests}

The authors declare that they have no competing interests.

Received: 17 December 2015 Accepted: 12 April 2016

Published online: 23 April 2016

\section{References}

Adouom OA (2009) Determination of toxicity levels of some savannah plants using brine shrimp test (BST). Bayero J Pure Appl Sci 2:135-138

Arcanjo DDR, Albuquerque ACM, Melo-Neto B, Santana LCLR, Medeiros MGF, Citó AMGL (2012) Bioactivity evaluation against Artemia salina L. each of medicinal plants used in Brazilian northeastern folk medicine. Braz J Biol 72:505-509

Biradi M, Hullatti K (2014) Screening of Indian medicinal plants for cytotoxic activity by brine shrimp lethality (BSL) assay and evaluation of their tota phenolic content. Drug Dev Ther 5:139-144

Bussmann RW, Malca G, Glenn A, Sharon D, Nilsen B, Parris B, Dubose D, Ruiz D, Saleda J, Martinez M, Carillo L, Walker K, Kuhlman A, Townesmith A (2011) Toxicity of medicinal plants used in traditional medicine in northern Peru. J Ethnopharm 137:121-140

Dietrich G, Dolan MC, Peralta-Cruz J, Schmidt J, Piesman J, Eisen RJ, Karchesy JJ (2006) Repellent activity of fractionated compounds from Chamaecyparis nootkatensis essential oil against Nymphal Ixodes scapularis (Acari: Ixodidae). J Med Entomol 43:957-961

Dolan MC, Dietrich G, Panella NA, Montenieri JA, Karchesy JJ (2007) Biocidal activity of three wood essential oils against Ixodes Scapularis (Acari: Ixodidae), Xenopsylla cheopis, and Aedes aegypti. J Econ Entomol 100:622-625

Dolan MC, Jordan RA, Schulze TL, Schulze CJ, Manning MC, Ruffolo D, Schmidt JP, Piesman J, Karchesy JJ (2009) Ability of two natural products, nootkatone and carvacrol, to suppress Ixodes scapularis and Amblyomma americanum (Acari: Ixodidae) in a lyme disease endemic area of New Jersey. J Econ Entomol 102:2316-2324

Forlines DR, Tavenner T, Malan JCS, Karchesy JJ (1992) Plants of the Olympic coastal forests: ancient knowledge of materials and medicines and future heritage. Basic Life Sci 59(Plant Polyphenols):767-782

Franklin JF, Dyrness CT (1988) Natural vegetation of Oregon and Washington USDA Forest Service Oregon State University Press, Corvallis

Gadir SA (2012) Assessment of bioactivity of some Sudanese medicinal plants using brine shrimp (Artemia salina) lethality assay. J Chem Pharm Res 4:4148-5145

Gunther E (1973) Ethnobotany of western Washington. Univ. Washington Press, Seattle

Harwig J, Scott PM (1971) Brine shrimp (Artemia salina L.) larvae as a screening system for fungal toxins. Appl Microbiol 21:1011-1016

Horgen FD, Edrada RA, de los Reyes G, Agcaoili F, Madulid DA, Wongpanich V Angerhofer CK, Pezzuto JM, Soejarto DD, Farnsworth NR (2001) Biological screening of rain forest plot trees from Palawan Islant (Philippines). Phytomedicine 8:71-81

Johnston WH, Karchesy JJ, Constantine GH, Craig AM (2001) Antimicrobial activity of some Pacific Northwest woods against anaerobic bacteria and yeasts. Phytother Res 15:586-588

Khatun A, Rahman M, Haque T, Rahman M, Akter M, Akter S, Jhumur A (2014) Cytotoxicity potentials of eleven Bangladeshi medicinal plants. The Sc World J. Article ID 913127, p 7. doi:10.1155/2014/913127

Krishnarajua AV, Raoa TVN, Sundararajua D, Vanisreeb M, Tsayb H-S, Subbarajua GV (2005) Assessment of bioactivity of Indian medicinal plants using brine shrimp (Artemia salina) lethality assay. Int J Appl Sci Eng 3:125-134
Manter DK, Karchesy JJ, Kelsey RG (2006) The sporidical activity of yellow-cedar heartwood, essential oil and wood constituents towards Phytophthora ramorum in culture. For Pathol 36:297-308

Manter DK, Kelsey RG, Karchesy JJ (2007) Antimicrobial activity of extractable conifer heartwood compounds toward Phytophthora ramorum. J Chem Ecol 33:2133-2147

McAllister JC, Adams MR (2010) Mode of action for natural products isolated from essential oils of two trees is different from available mosquito adulticides. J Med Entomol 47:1123-1126

McLaughlin JL, Chang CJ, Smith DL (1991) "Bench-top" bioassays for the discovery of bioactive natural products: an update. In: Rahman A (ed) Studies in natural product chemistry, vol 9. Elsevier, Amsterdam, pp 383-409

McLaughlin JL, Rogers LL, Anderson JE (1998) The use of biological assays to evaluate botanicals. Drug Inform J 32:513-524

Meyer BN, Ferrigini RN, Putnam JE, Jacobsen LB, Nichols DE, McLaughlin JL (1982) Brine shrimp: a convenient general bioassay for active plant constituents. Planta Med 45:31-35

Michael AS, Thompson CG, Abramovitz M (1956) Artemia salina as a test organism for bioassay. Science 123:464

Moore M (1993) Medicinal plants of the Pacific West. Red Crane Books, Santa $\mathrm{Fe}$

Moshi MJ, Innocent E, Magadula JJ, Otieno DF, Weisheit A, Mbabazi PK, Nondo RSO (2010) Brine shrimp toxicity of some plants used as traditional medicines in Kagera Region, northwestern Tanzania. Tanzania J Health Res 12:63-67

Nguta JM, Mbaria JM, Gakuya DW, Gathumbi PK, Kabasa JD, Kiama SG (2011) Biological screening of Kenyan medicial plants using A. salina L. (Artemiidae). Pharmacologyonline 2:458-478

Nguta JM, Mbaria JM, Gakuya DW, Gathumbi PK, Kabasa JD, Kiama SG (2012) Evaluation of acute toxicity of crude plant extracts from Kenyan biodiversity using brine shrimp, Artemia salina L. (Artemiidae). Open Conf Proc J 3:30-34

Oryema C, Ziraba RB, Odyek O, Omagor N, Opio A (2011) Phytochemical properties and toxicity to brine shrimp of medicinal plants in Erute county, Lira district, Uganda. J Med Plants Res 5:5450-5457

Panella NA, Dolan MC, Karchesy JJ, Xiong Y, Peralta-Cruz J, Khasawneh M, Montenieri JA, Maupin GO (2005) Use of novel compounds for pest control: insecticidal and acaricidal activity of essential oil components from heartwood of Alaska yellow cedar (Chamaecyparis nootkatensis). J Med Entomol 42:352-358

Pimentel AB, Pizzolatti MG, Costa IM (2002) An application of the brine shrimp bioassay for general screening of Brazilian medicinal plants. Acta Farm Bonaerense 21:175-178

Rahman MS, Begum B, Chowdhury R, Rahman KM, Rashid MA (2008) Preliminary cytotoxicity screening of some medicinal plants of Bangladesh. Dhaka Univ J Pharm Sci 7:47-52

Ravikumar AR, Madgaonkar V, Venkatesha RT, Bharathi R, Murthy VK (2014) Potential cytotoxic drug effects of secondary metabolites derived from selected medicinal plants of Savanadurga forest in Karnataka. Int J Pharm Pharm Sci 6:238-241

Rizwana JN, Nazlina I, Razehar ARM, Noraziah AZS, Ling CY, Muzaimah SAS, Farina AH, Yaacob WA, Ahmad IB, Din LB (2010) A survey on phytochemical and bioactivity of plant extracts from Malaysian forest reserves. J Med Plants Res 4:203-210

Soonthornchareonnon N, Wiwat C, Chuakul W (2012) Biological activities of medicinal plants from mangrove and beach forests. Mahidol Univ J Pharm Sci 39:9-18

Ved CH, More NS, Bharate SS, Bharate SB (2010) Cytotoxicity screening of selected Indian medicinal plants using brine-shrimp lethality bioassay. Adv Nat Appl Sci 4:389-395 